\title{
Optical coherence tomography and fundus autofluorescence imaging study of chorioretinal atrophy involving the macula in Alagille syndrome
}

This article was published in the following Dove Press journal:

Clinical Ophthalmology

5 September 2012

Number of times this article has been viewed

\author{
Shinji Makino \\ Yuko Ohkubo \\ Hironobu Tampo \\ Department of Ophthalmology, \\ Jichi Medical University, Shimotsuke, \\ Tochigi, Japan
}

\begin{abstract}
We report the first case in the literature of chorioretinal atrophy involving the macula in an 11-year-old girl with Alagille syndrome, as examined by optical coherence tomography, and fundus autofluorescence imaging. Funduscopy revealed diffuse choroidal hypopigmentation with increased visibility of the choroidal vessels and symmetric, well circumscribed macular discoloration. Anomalous oblique configuration of the optic disc and peripapillary tortuous vessels were also detected. Optical coherence tomography demonstrated decreased retinal thickness, especially the outer retinal layer, and the photoreceptor inner segment/outer segment junctions were irregular and discontinued, corresponding to macular discoloration. Fundus autofluorescence imaging clearly defined hypofluorescent areas in the peripapillary regions that extended along the macula and had a sleep mask appearance. We suggest that transient hypovitaminosis due to Alagille syndrome early in life might contribute to the retinal degeneration seen in this case.
\end{abstract}

Keywords: Alagille syndrome, chorioretinal atrophy, optical coherence tomography, fundus autofluorescence imaging, hypovitaminosis

\section{Introduction}

Alagille syndrome is clinically defined by neonatal cholestatic jaundice with intrahepatic bile duct hypoplasia associated with additional findings, including a characteristic facies, peripheral pulmonary artery stenosis, butterfly-like vertebral anomalies, and ocular abnormalities. Ocular findings include posterior embryotoxon, iris abnormalities, optic disc anomalies, and fundus changes (irregular pigmentation at the level of the retinal pigment epithelium, diffuse hypopigmentation, and punched out chorioretinal atrophy). ${ }^{1,2}$

To our knowledge, there are no cases in the literature presenting with Alagille syndrome and chorioretinal atrophy involving the macula, as examined by optical coherence tomography (OCT) and fundus autofluorescence. Herein, we report OCT and fundus autofluorescence changes in a patient with this syndrome.

\section{Case report}

An 11-year-old girl with Alagille syndrome was referred to the Jichi Medical University Hospital before a liver biopsy during observation after liver transplantation. Liver transplantation was performed at the age of 20 months, and left pulmonary artery balloon dilation was performed at the age of 8 years. She had an unusual triangular facies characterized by a broad overhanging forehead, deep-set hyperteloric eyes, and a small
Correspondence: Shinji Makino

33 I I-I Yakushiji, Shimotsuke,

Tochigi 329-0498, Japan

Tel $+8 I 285587382$

Fax +8I 285448365

Email makichan@jichi.ac.jp 
pointed chin. She had no problems with cognitive functioning. On ophthalmic examination, best-corrected visual acuity in the right eye was $12 / 20$ with $-6.25 \mathrm{D}-1.0 \mathrm{D} \times 90^{\circ}$, and in the left eye was $8 / 20$ with $-4.25 \mathrm{D}-2.0 \mathrm{D} \times 90^{\circ}$. The patient was orthophoric and showed normal ocular movements. On slit lamp examination, posterior embryotoxon was absent in both eyes. The lens was clear in both eyes. Ocular pressures were normal. Funduscopy showed diffuse choroidal hypopigmentation with increased visibility of the choroidal blood vessels and symmetric well circumscribed macular discoloration (Figure 1). Anomalous oblique configuration of the optic disc and peripapillary tortuous vessels were also detected.

Decreased retinal thickness was seen on OCT (RS-3000, Nidek, Japan). On the basis of macular ETDRS (Early Treatment Diabetic Retinopathy Study) sectors, thickness of the center (central fovea), inner ring (1-3 mm from the central fovea) and outer ring (3-6 mm from the central fovea) were $222 \mu \mathrm{m}, 254.8 \mu \mathrm{m}$, and $241.0 \mu \mathrm{m}$, respectively, in the right eye. In the left eye, these were $228 \mu \mathrm{m}, 246.8 \mu \mathrm{m}$, and 230.3 $\mu \mathrm{m}$, respectively. In particular, the outer retinal (nuclear) layer and the photoreceptor inner segment/outer segment junctions were irregular and discontinuous, corresponding to macular discoloration (Figures 2 and 3). Foveal architecture was preserved in both eyes with thinning. In high-magnification OCT images, the border between the chorioretinal atrophy and the hypopigmented area of the macula was clearly defined. The outer retinal thickness in the chorioretinal atrophic area was thinner than that in the hypopigmented area. Fundus autofluorescence imaging (Heidelberg Retina Angiograph 2, Heidelberg, Germany) showed hypofluorescent areas in the peripapillary regions extending along the macula corresponding to the chorioretinal atrophy. These had an appearance similar to a sleep mask (Figure 4). A mottled pattern of hyperautofluorescent areas was also detected in the macula. Visual field testing with Goldmann perimetry only showed enlargement of the blind spots in both eyes.
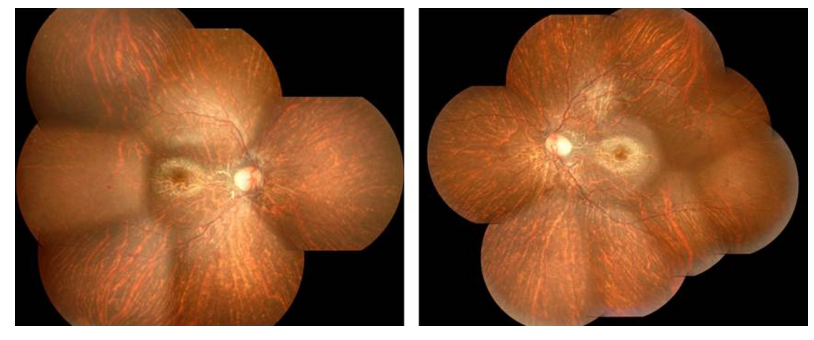

Figure I Right and left fundus photographs showing diffuse choroidal hypopigmentation with increased visibility of the choroidal vessels and symmetric well circumscribed macular discoloration.
Color vision was normal with Ishihara color plate tests. Serum vitamin A and vitamin E levels were 94 (normal range 97-316) IU/dL and 0.51 (normal range 0.75-1.41) mg/dL, respectively.

\section{Discussion}

In the largest series of patients with Alagille syndrome, Hingorani et $\mathrm{al}^{1}$ evaluated 22 patients with the condition. The most common ocular abnormalities in Alagille syndrome were posterior embryotoxon (95\%), iris abnormalities (45\%), diffuse fundus hypopigmentation (57\%), speckling of the retinal pigment epithelium (33\%), and optic disc anomalies $(76 \%) .{ }^{1}$ In studying retinal changes in Alagille syndrome, Hingorani et $\mathrm{al}^{1}$ reported that irregular pigmentation at the level of the retinal pigment epithelium, most often manifested as a speckling or granularity of the retinal pigment epithelium, was distributed diffusely in the mid peripheral/peripheral zone. ${ }^{1}$ The few reports concerning macular changes in Alagille syndrome describe symmetric, well circumscribed, horizontally oval areas of speckled reddish brown macular discoloration, ${ }^{2}$ pigment clumping in the macula, ${ }^{3}$ and circumpapillary geographic chorioretinal atrophy with half-moon shapes involving the macular area. ${ }^{4}$ However, chorioretinal atrophy involving the macular area is rare. In this case, diffuse choroidal hypopigmentation with increased visibility of the choroidal vessels and a sleep masklike hypofluorescent area corresponding to chorioretinal atrophy as examined by fundus autofluorescence were detected. To our knowledge, there are no similar cases in the literature of Alagille syndrome presenting a chorioretinal atrophy involving the macula with a sleep mask appearance as examined by OCT and fundus autofluorescence.

A possible explanation for chorioretinal atrophy in Alagille syndrome is failure of absorption of fat-soluble vitamins. Romanchuk et $\mathrm{al}^{5}$ suggested that failure of fatsoluble vitamin absorption plays a major role in the evolution of retinopathy in Alagille syndrome. The retinoid cycle is a vitamin A-linked metabolic circuit which occurs between the outer segment of the photoreceptor and the retinal pigment epithelium to maintain visual function. Low serum vitamin E levels have been well documented in children with malabsorption due to cholestatic liver diseases. ${ }^{6}$ The role of vitamin $\mathrm{E}$ in vision is less clear, but it is believed to have an important protective function as a lipid antioxidant for the extremely high concentrations of polyunsaturated fatty acids found in the outer segments. Increases in the number of lipofuscin granules in the cytoplasm of retinal pigment epithelium in vitamin E-deficient rats were observed. ${ }^{7}$ 

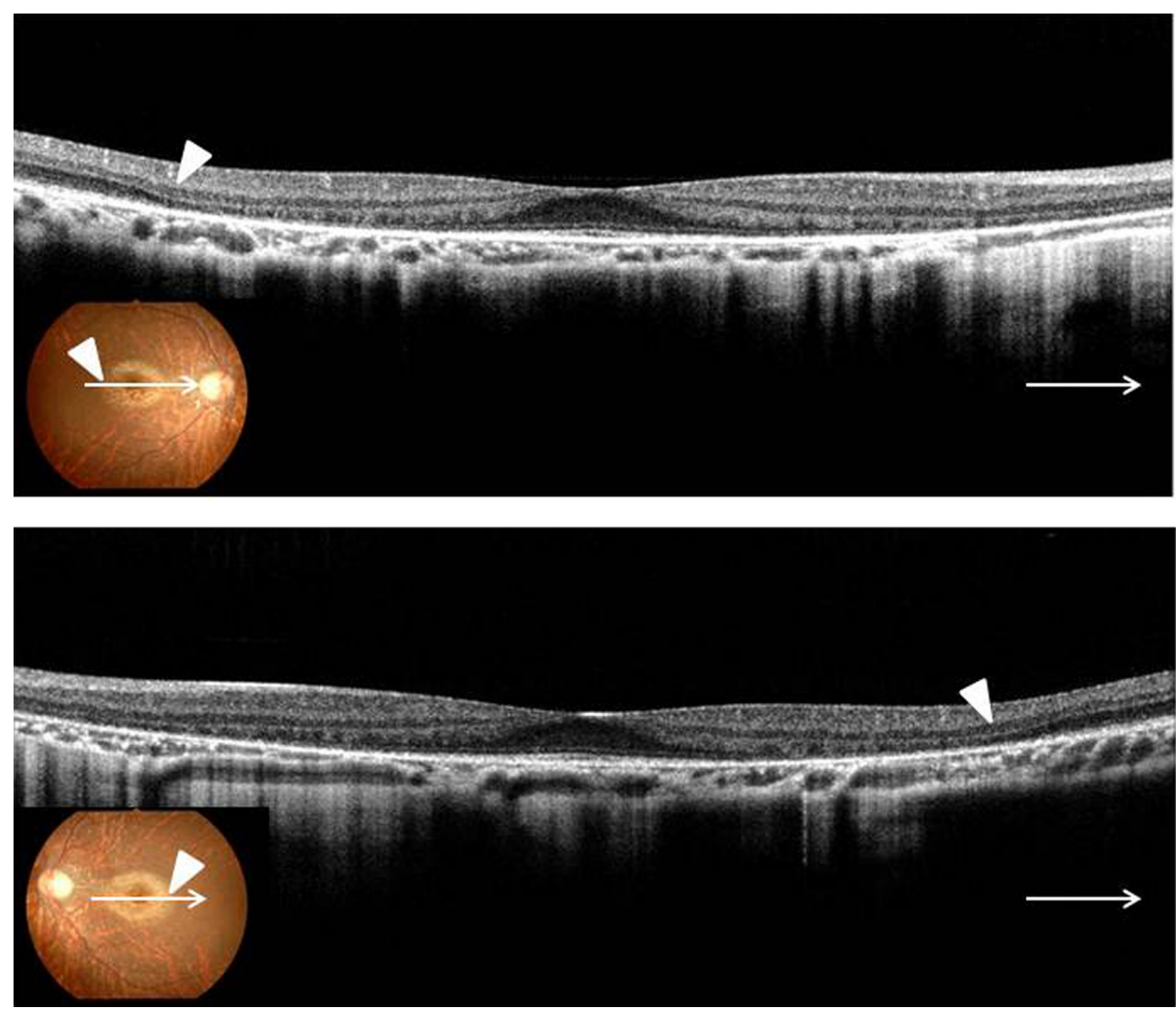

Figure 2 Right and left horizontal optical coherence tomography scan showed decreased retinal thickness.

Notes: The outer retinal layer and the photoreceptor inner segment/outer segment junction were irregular and discontinuous, corresponding to macular discoloration. An arrowhead indicates the temporal border of macular discoloration. Foveal architecture was preserved in both eyes, with thinning.
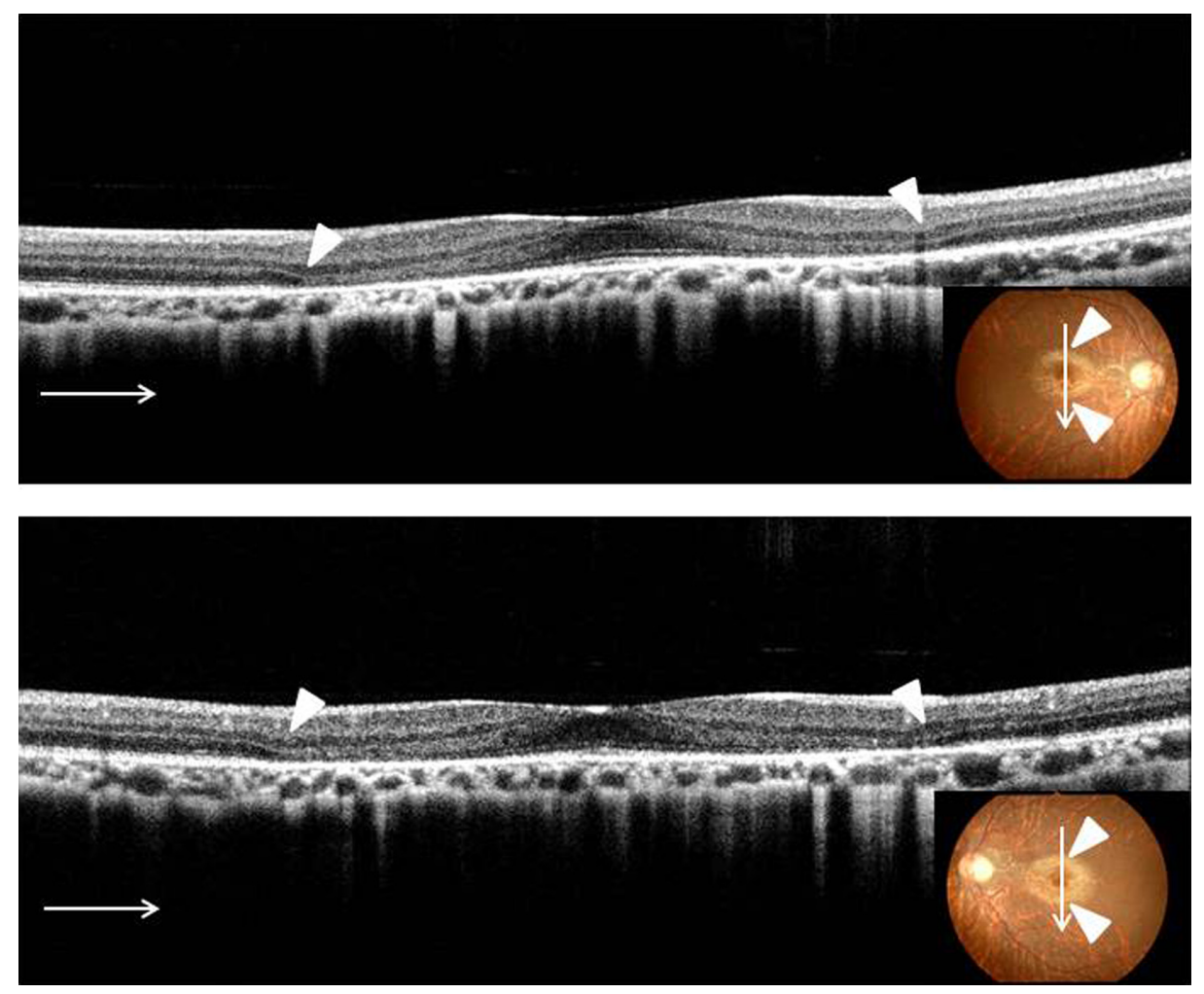

Figure 3 Right and left vertical optical coherence tomography scan had the same findings as horizontal optical coherence tomography scans. Note: Arrowheads indicate upper and lower borders of macular discoloration. 

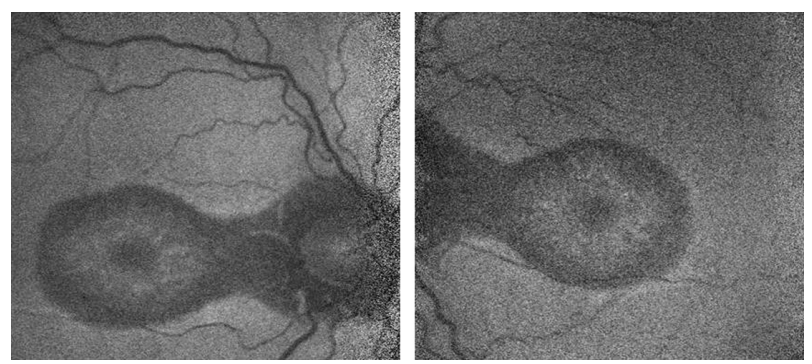

Figure 4 Right and left fundus autofluorescence imaging showing clearly defined hypofluorescent areas in the peripapillary regions that extend along the macula, with a sleep mask appearance.

Note: A mottled hyperautofluorescent area is also detected in the macula.

The retinal pigment epithelium cells of the peripheral and equatorial zone contain large numbers of lipofuscin granules within their cytoplasm. ${ }^{8}$ Thus, both of these vitamins are believed to be important in maintaining the integrity of the photoreceptor outer segments and the retinal pigment epithelium cells.

Fundus autofluorescence has been investigated in various fundus diseases associated with changes in the retinal pigment epithelium. Fundus autofluorescence is presumed to derive from lipofuscin in retinal pigment epithelium cells and to represent metabolic activity of retinal pigment epithelium involving turnover of photoreceptor outer segments. Hypofluorescence is thought to correspond to areas of decreased metabolism resulting from photoreceptor and/or retinal pigment epithelium atrophy, and has been used as a marker of the integrity of the retinal pigment epithelium/photoreceptor complex. The peculiar findings in this patient were abnormalities of the inner segment/outer segment junction and the outer segments of photoreceptors, suggestive of loss of structural integrity of the photoreceptors. The mottled lesions in this patient could have consisted of partially preserved photoreceptor layers.
Therefore, we suggest that transient hypovitaminosis due to Alagille syndrome early in life might contribute to the retinal degeneration seen in this case.

Finally, anatomic evidence of retinal degeneration induced by vitamin $\mathrm{A}$ or $\mathrm{E}$ deficiencies suggests that the structural disruption of photoreceptors is more advanced in cones and most pronounced in the macula, with lesser involvement of the peripheral retina. ${ }^{9}$ However, it is not clear whether our case represents a congenital or acquired change due to Alagille syndrome. Further studies with additional cases are necessary to answer this question.

\section{Disclosure}

No conflicts of interest are declared in relation to this paper.

\section{References}

1. Hingorani M, Nischal KK, Davies A, et al. Ocular abnormalities in Alagille syndrome. Ophthalmology. 1999;106:330-337.

2. Brodsky MC, Cunniff C. Ocular anomalies in Alagille syndrome (arteriohepatic dysplasia). Ophthalmology. 1993;100:1767-1774.

3. Wells KK, Pulido JS, Judisch GF, Ossoinig KC, Fisher TC, LaBrecque DR. Ophthalmic features of Alagille syndrome (arteriohepatic dysplasia). J Pediatr Ophthalmol Strabismus. 1993;30:130-135.

4. Bidaguren A, Blanco A, Gibelalde A, Irigoyen C. Progressive geographic chorioretinopathy associated with Alagille syndrome. Arch Soc Esp Oftalmol. 2007;82:513-516.

5. Romanchuk KG, Judisch GF, LaBrecque DR. Ocular findings in arteriohepatic dysplasia (Alagille's syndrome). Can JOphthalmol. 1981;16: 94-99.

6. Rosenblum JL, Keating JP, Prensky AL, Nelson JS. A progressive neurologic syndrome in children with chronic liver disease. $N$ Engl J Med. 1981;304:503-508.

7. Robinson WG Jr, Kuwabara T, Bieri JG. Vitamin E deficiency and the retina: photoreceptor and pigment epithelial changes. Invest Ophthalmol Vis Sci. 1979;18:683-690.

8. Johnson BL. Ocular pathologic features of arteriohepatic dysplasia (Alagille syndrome). Am J Ophthalmol. 1990;110:504-512.

9. Hayes KC. Retinal degeneration in monkeys induced by deficiencies of vitamin E or A. Invest Ophthalmol Vis Sci. 1974;13:499-510.
Clinical Ophthalmology

\section{Publish your work in this journal}

Clinical Ophthalmology is an international, peer-reviewed journal covering all subspecialties within ophthalmology. Key topics include: Optometry; Visual science; Pharmacology and drug therapy in eye diseases; Basic Sciences; Primary and Secondary eye care; Patient Safety and Quality of Care Improvements. This journal is indexed on Submit your manuscript here: http://www.dovepress.com/clinical-ophthalmology-journal

\section{Dovepress}

PubMed Central and CAS, and is the official journal of The Society of Clinical Ophthalmology (SCO). The manuscript management system is completely online and includes a very quick and fair peer-review system, which is all easy to use. Visit http://www.dovepress.com/ testimonials.php to read real quotes from published authors. 